This is the final peer-reviewed accepted manuscript of:

Fabiani, D., Zaccaria, M., Zucchelli, A., Belcari, J., Bocchi, O.

Electrospun nanofibers with piezoelectric properties

(2015) 33rd Electrical Insulation Conference, EIC 2015, art. no. 7223520, pp. 197200

The final published version is available online at:

https://doi.org/10.1109/ICACACT.2014.7223520

Rights / License:

(C)2015.This article may be used for non-commercial purposes in accordance with Wiley Terms and Conditions for Use of Self-Archived Versions. 


\title{
Electrospun Nanofibers with Piezoelectric Properties
}

\author{
Davide Fabiani, Marco Zaccaria \\ Dept. of Electrical Electronics and Information \\ Engineering \\ Alma Mater Studiorum University of Bologna \\ Bologna, Italy \\ davide.fabiani@unibo.it
}

\author{
Andrea Zucchelli, \\ Dept. of Industrial Engineering \\ Alma Mater Studiorum University of Bologna \\ Bologna, Italy
}

\author{
Juri Belcari and Oliviero Bocchi \\ CIRI - Advanced Applications in Mechanical Engineering and Materials Technology \\ Alma Mater Studiorum University of Bologna \\ Bologna, Italy
}

\begin{abstract}
This paper presents a preliminary comparison between the piezoelectric behavior of PVdF electrospun nanofibrous membranes and a commercial PVdF film. XRD measurements showed that the $\beta$ phase of the realized electrospun PVdF membranes is much larger than that of the PVdF film here considered. Consequently, the nanofibrous membranes show a significant increase of the electrical response associated with a mechanical impulsive stress applied to the sample, with respect to that provided by film specimens. In particular, the electric signal per unit weight is much larger for the nanofibrous specimens, i.e. a few orders of magnitude larger than that of commercial flat specimens. This behavior could be of interest, e.g, in the field of impact sensors and energy harvesting.
\end{abstract}

Keywords -Piezoelectric materials; nanofibers; electrospinning; sensors; energy harvesting.

\section{INTRODUCTION}

Electrospinning is a quite simple technique, which allows materials to be produced in the form of continuous polymer fibers, having diameters ranging from tens of nanometers to some micrometers. This process is based on the electrostatic charging of a polymer jet in liquid phase, e.g. by solution or melt [1]. The nanofibrous materials obtained through this process can have either random non-woven or aligned fiber pattern, depending on collector geometry, experimental apparatus and operating conditions $[1,2]$. Several application fields can be envisaged for such nanofibrous mats, from tissue engineering to water and air filtering, and, last but not least, electronic sensors and energy storage devices [3, 4].

It is well known in literature that some types of PVdF and PVdF co-polymers show piezoelectric properties in peculiar crystallinity phase ( $\beta$ phase) [5]. Usually, PVdF is manufactured through conventional production techniques in form of laminated film or through solvent casting. Then, in order to maximize the $\beta$ phase, quite complex techniques must be implemented, e.g. by means of particular annealing procedures and/or mechanical stretching followed by electric polarization using high electric field $[5,6]$.
Only few studies, on the contrary, have been performed on fibrous PVdF, showing piezoelectric behavior, e.g. obtained through electrospinning [7-10]. The electrospinning technique, in fact, provides at the same time mechanical stretching of the polymer chains and electrical poling, thus increasing, in principle, piezoelectric $\beta$ phase. Electrospun membranes are thin, lightweight and more flexible compared to conventional piezoelectric materials and they can be easily manufactured in different shapes, with random or aligned fiber patterns. Electrospun nanometric fibers can be implemented as spun or deposited on solid substrates showing peculiar properties, e.g. due to their wide surface-to-volume ratio, and multifunctional or smart behavior at the nanoscale: they could become suitable as energy harvesting devices or sensors.

In this work, preliminary measurements to characterize the piezoelectric behavior of $\mathrm{PVdF}$ electrospun nanofibrous membranes are shown and a first comparison to the piezoelectric features obtained on a commercial PVdF film is carried out through XRD measurements and repetitive mechanical impulsive tests.

\section{MATERIALS AND METHODS}

PVdF Solef 6008 and PVdF-TrFe were kindly provided by Solvay Specialty Polymers (Bollate, Italy). A commercial piezoelectric film of PVdF (Goodfellow), about $500 \mu \mathrm{m}$ thick, was tested after silver ink metallization.

A laboratory electrospinning machine (Spinbow Lab Unit, Spinbow S.r.l., Italy) was used to produce electrospun mats. The machine was equipped with a multi-jet linear sliding apparatus having 4 needles, a rotating drum collector and a syringe pump (KDScientific 200 series). The process was done at room temperature and relative humidity $30-40 \%$. Membranes about $30 \times 40 \mathrm{~cm}^{2}$ were fabricated for each type of polymer solution, having $40-50 \mu \mathrm{m}$ thickness measured using a digital micrometer (Borletti MDE1, resolution $0.001 \mathrm{~mm}$ ). A schematic picture of the electrospinning process is reported in Figure 1. Fiber morphology was observed through a scanning electron microscope (PHENOM PROX Desktop SEM). 


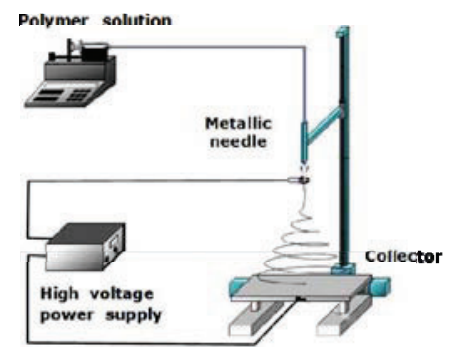

Fig. 1. Electrospinning process schematic.

PVdF 6008 was dissolved at a concentration of $15 \% \square \mathrm{w} / \mathrm{v}$ in Acetone (Ac):Dimethyl Sulfoxide (DMSO) (70:30, v/v). Good fiber morphology without defects is shown in Figure 2.

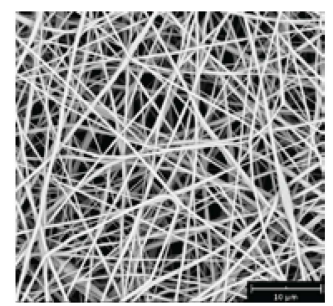

Fig. 2. SEM image of electrospun PVdF 6008; scale bar $=10 \mu \mathrm{m}$.

Different polymeric solutions of PVdF-TrFe were investigated with the aim of optimizing fiber morphology. For this reason different solvent systems were tested: PVdF-TrFe was dissolved (i) at $25 \% \mathrm{w} / \mathrm{v}$ in $100 \%$ Dimethyl Formamide (DMF), (ii) at 20\% w/v in Ac:DMSO (70:30, v/v) and (iii) at $17.5 \% \mathrm{w} / \mathrm{v}$ in DMF:Ac $(56: 44, \mathrm{v} / \mathrm{v})$. The comparison between fibers obtained from the three solutions is reported in Figure 3. It is possible to observe that $100 \%$ DMF leads to the formation of bead-like defects (Figure 3A), which worsen mechanical properties of the realized membrane. Good quality fibers without defects were obtained using the other solvent systems (Figure 3B and 3C). In particular, DMF:Ac solvent system led to fiber diameters of $350 \pm 100 \mathrm{~nm}$ (see Figure 3C), while fibers electrospun by Ac:DMSO solution showed diameters of $820 \pm 100 \mathrm{~nm}$ (see Figure 3B). Therefore, mats obtained by DMF:Ac solution were chosen for electro-mechanical tests, due to thinner fiber diameters. Indeed, sub-micrometric fibers, thanks to their high surface to volume ratio, are suitable for applications in the nanotechnology field.
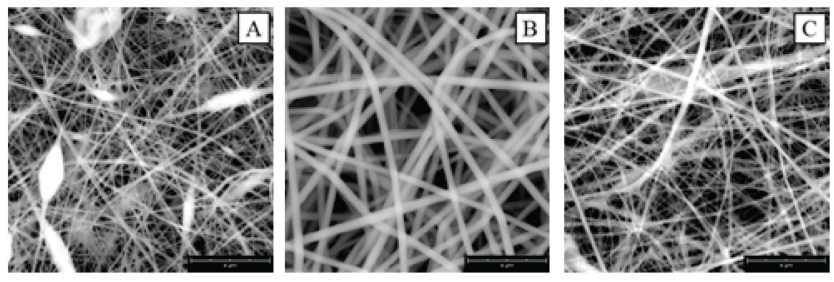

Fig. 3. SEM images of electrospun PVdF-TrFe obtained by different solvent systems: DMF (A), Ac:DMSO (B), DMF:Ac (C); scale bar $=8 \mu \mathrm{m}$.

$\mathrm{X}$-ray spectroscopy was implemented to detect crystalline phases of the PVDF. X-ray diffraction patterns were obtained with $\mathrm{CuK} \alpha$ radiation in reflection mode by means of an X'Pert PANalytical diffractometer equipped with a fast $X^{\prime}$ Celerator detector, step $0.1^{\circ}, 150 \mathrm{~s} / \mathrm{step}$.
Piezoelectric samples of $30 \times 40 \mathrm{~mm}^{2}$ were subjected to mechanical impulses given at different frequencies, ranging from $0.5 \mathrm{~Hz}$ to $5 \mathrm{~Hz}$, through an electromagnetic piston $(10$ $\mathrm{mm}$ diameter), controlled with National Instruments LabVIEW $^{\mathrm{TM}}$. Two different masses were connected to the system thus the total weight of the piston was $350 \mathrm{~g}$ and $500 \mathrm{~g}$, respectively. Every test was carried out acquiring at least ten signals at each frequency for any weight. Aluminum foils deposited on the electrospun specimen surfaces were used as electrodes and connected to a digital oscilloscope (Tektronix DPO 5034) in order to detect and record the electric signals generated by mechanical impulses. The experimental apparatus shown in Figure 4 was used for both electrospun samples and commercial films.

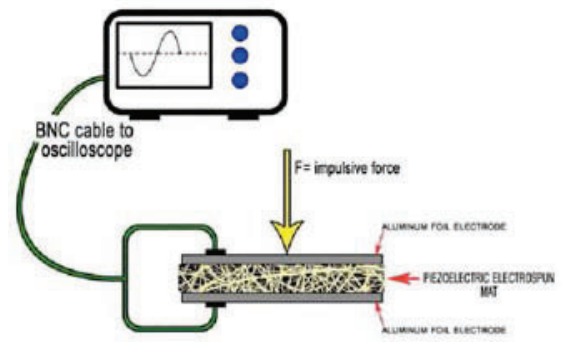

Fig. 4. Experimental apparatus of the electro-mechanical tests carried out on electrospun samples.

\section{RESULTS}

The production of electrospun piezoelectric membranes was successfully carried out and samples were tested without any type of post-treatment, such as mechanical stretching or electrical poling.

Figure 5 shows the XRD spectra of the nanofibrous PVdFTrFe specimen and PVdF commercial film. As can be seen, both materials show evidences of $\beta$-phase, which is indicated by the peak around $20^{\circ}$. The commercial PVdF film, however, exhibits also $\alpha$-phase, which has a very weak piezoelectric behavior, corresponding to the peaks at $17.5^{\circ}$ and $26^{\circ}$ [5]. It is worth noting that these peaks are absent in the electrospun nanofibrous specimen, indicating that $\beta$-phase is much larger than that shown by the commercial film. This feature will positively affect also the piezoelectric behavior of the two materials, as will be shown in the following.

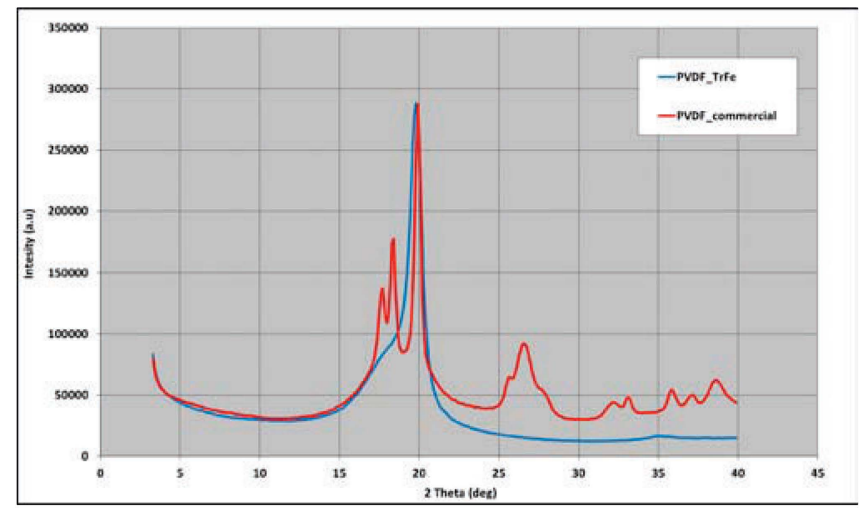

Fig. 5. XRD spectra of electrospun PVdF-TrFe and commercial PVdF film. 
Highly reproducible waveforms were detected from the impulsive mechanical tests, as can be observed in Figure 6, which displays electrical response to $350 \mathrm{~g}$ stress at $4 \mathrm{~Hz}$ for PVdF 6008 and PVdF-TrFe. From waveform analysis, peak-topeak voltage spectra can be also obtained and are displayed in Figures 7 and 8 for PVdF-TrFe and PVdF 6008, respectively. Since all the samples tested in this work provided voltage signals having large amplitude, i.e. in the Volt range, the use of an amplifier to process the signal could be avoided. Looking at Figures 7 and 8 , it can be clearly observed that electrical signals related to $\mathrm{PVdF}-\mathrm{TrFe}$ samples are significantly larger than those relevant to PVdF 6008. This behavior could be explained considering that PVdF 6008 is a homopolymer having almost linear chains while PVdF-TrFe is a co-polymer, specifically designed to enhance piezoelectric behavior [10]. The electric response with frequency of PVdF-TrFe shows that the higher the frequency, the larger the peak-to-peak voltage detected. On the contrary, PVdF 6008 shows that voltage signal increases, as the frequency rises, up to a given value, beyond which a sudden decrease is observed. This behavior could be explained considering that the co-polymer, having a structure characterized by highly polar branch chains, is able to provide good piezoelectric response at higher frequencies with respect to PVdF 6008. It is worth noting that for the $500 \mathrm{~g}$ mechanical stress on PVdF 6008 the piezoelectric response saturates at frequencies larger than $2 \mathrm{~Hz}$. For the $350 \mathrm{~g}$ mechanical stress an increasing trend is seen up to $4 \mathrm{~Hz}$, beyond which the signal drops. It can be speculated that for $350 \mathrm{~g}$ mechanical stress, saturation phenomenon could likely occur at a higher frequency, exceeding the range here investigated.
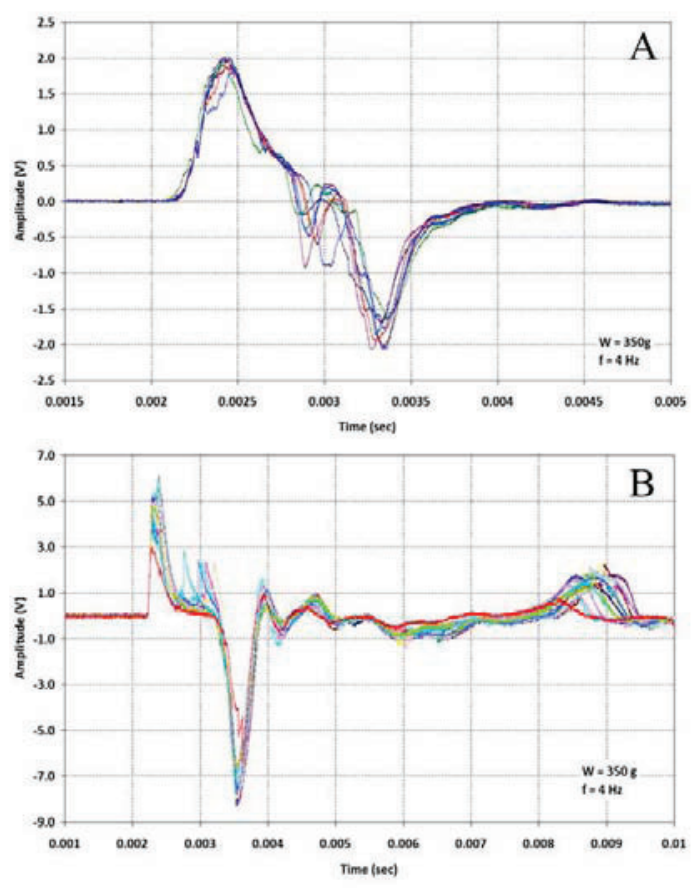

Fig. 6. Plots reporting overlapped waveforms obtained from repetitive tests done with a stress of $350 \mathrm{~g}$ at $4 \mathrm{~Hz}$ on electrospun PVdF 6008 (A) and PVdF$\operatorname{TrFe}(\mathrm{B})$.

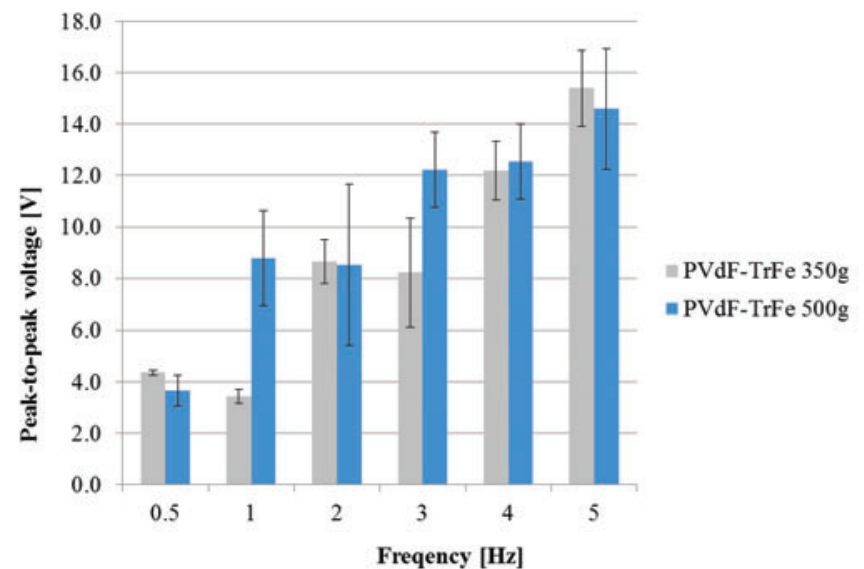

Fig. 7. Voltage response (peak-to-peak value) of electrospun PVdF-TrFe as a funcion of frequency.

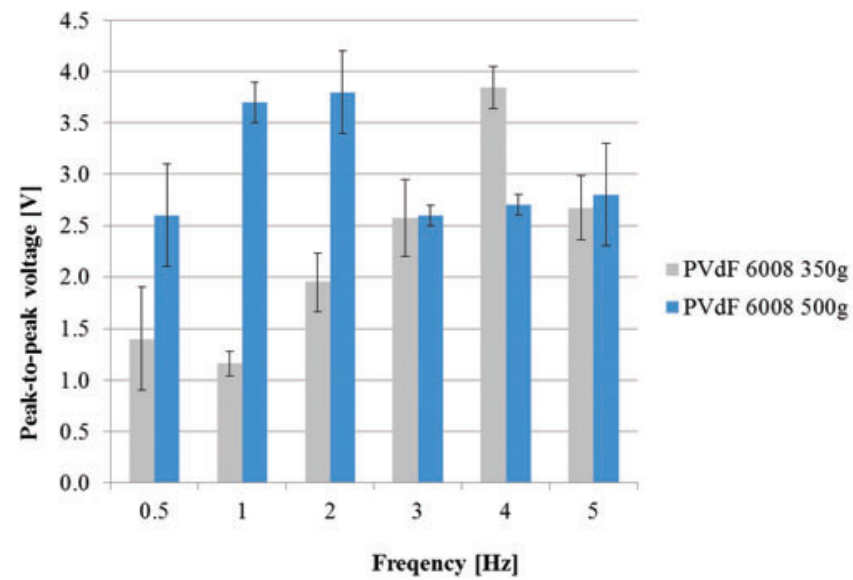

Fig. 8. Voltage response (peak-to-peak value) of electrospun PVdF 6008 as a funcion of frequency.

In order to assess the effect of repetitive mechanical stress on material conditions, SEM images of PVdF-TrFe after several impacts given by the electromagnetic piston $(500 \mathrm{~g})$ are reported in Figure 9. In particular, Figure 9A displays a large specimen region showing two different zones, one clearly affected by mechanical stress and another one where the typical fibrous structure is still present. Zooming in the impact zone (Figure 9B), it is clearly evidenced that the typical fibrous pattern of electrospun mats (see Figure 3C) radically changes into a film-type structure. Indeed, repeated impacts applied to the fibrous material press the mat, reducing the air gaps between fibers and, consequently, compacting the fibrous sample into a less porous material. In general, such phenomenon is not a symptom of material degradation, since electric response may remain quite large also for higher impact rate (see Figure 7), but affects permanently sample morphology, thus modifying piezoelectric signals obtained after several mechanical impulses. Nevertheless, sometimes a significant degradation of the material is observed after several impacts, particularly in thinner specimens, leading to electrode short-circuiting in short times. This can explain also the large confidence intervals shown in Figures 7 and 8 . It has to be pointed out, however, that particularly harsh mechanical stress conditions have been here used to investigate piezoelectric 
behavior. With the aim of using these nanofibrous specimens as impact sensors or in energy storage applications, permanent changes in the polymer morphology should be avoided, applying lower mechanical stresses to piezoelectric fibers.

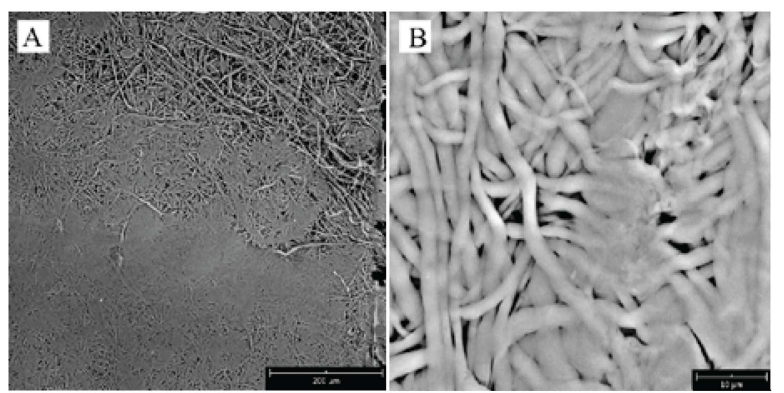

Fig. 9. SEM image of electrospun PVdF-TrFe after several mechanical impulses generated by the piston: transition from fiber-like to film-like strcuture (A) and detail of impact zone (B); scale bar $=200 \mu \mathrm{m}(\mathrm{A})$ and $10 \mu \mathrm{m}$ (B).

A commercial PVdF film was tested with the aim of comparing voltage response to that of electrospun mats. Results are summarized in Table 1, which reports a selected frequency of testing, i.e. $2 \mathrm{~Hz}$. It is possible to highlight that the response of commercial piezoelectric is larger than that of electrospun PVdF 6008, in particular at $500 \mathrm{~g}$ stress. Electrospun PVdF$\mathrm{TrFe}$ shows values of peak-to-peak voltage much higher than commercial PVdF, i.e. about four times greater. This behavior can be explained considering that the piezoelectric effect is associated also with the so-called "domain wall motion" [11]. In this case, nanofibrous nature of electrospun material allows larger mobility of domain walls, leading to higher piezoelectric responses with respect to film materials. Furthermore, the most interesting issue coming from the experimental results is that a so large piezoelectric effect is obtained from a fibrous sample constituted of about $90 \%$ vol of air. Considering the measured average weight of an electrospun specimen and of a commercial PVdF film as about $30 \mathrm{mg}$ and $1 \mathrm{~g}$, respectively, the values of average specific piezoelectric response (in $\mathrm{V} / \mathrm{g}$ ) are reported in Table 2. It can be highlighted that specific response of electrospun $\mathrm{PVdF}$ is 1-2 orders of magnitude larger than commercial PVdF. These findings could allow sensors and/or harvesters having very low weight to be manufactured for high energy density applications.

\section{CONCLUSIONS}

The feasibility to realize highly sensitive electrospun piezoelectric materials has been investigated. The peculiar feature of electrospun materials, which do not need postprocessing treatments, provides an interesting advantage with respect to film-type materials, due to cost and time savings. Moreover, the industrial scale-up of this manufacturing technique could be effectively developed. Furthermore, piezoelectric behavior of electrospun mats can benefit from typical nanofiber properties, such as high mobility and surfaceto-volume ratio. Indeed, high porosity of the membrane, as well as the larger content of $\beta$ phase with respect to commercial films, allows fibers to provide a large piezoelectric response (in the Volt range) when mechanically stressed. Finally, flexibility and possibility of realizing tunable shapes are other interesting properties of such fibrous materials.

TABLE I. VOLTAGE RESPONCE OF ELECTROSPUN SAMPLES AND COMMERCIAL PVDF FILM STRESSED WITH 350 G AND 500 G AT 2 HZ.

\begin{tabular}{|c|c|c|}
\hline \multirow{2}{*}{ Sample } & \multicolumn{2}{|c|}{ Piezoelectric response (peak-to-peak) [V] } \\
\cline { 2 - 3 } & $\mathbf{3 5 0} \boldsymbol{g}$ & $\mathbf{5 0 0} \boldsymbol{g}$ \\
\hline $\begin{array}{c}\text { Commercial } \\
\text { PVdF film }\end{array}$ & $1.4 \pm 0.4$ & $2.3 \pm 0.5$ \\
\hline $\begin{array}{c}\text { Electrospun } \\
\text { PVdF 6008 }\end{array}$ & $2.0 \pm 0.3$ & $3.8 \pm 0.4$ \\
\hline $\begin{array}{c}\text { Electrospun } \\
\text { PVdF-TrFe }\end{array}$ & $8.7 \pm 0.9$ & $8.5 \pm 3.1$ \\
\hline
\end{tabular}

TABLE II. SPECIFIC VOLTAGE RESPONCE OF ELECTROSPUN SAMPLES AND COMMERCIAL PVDF FILM STRESSED WITH $350 \mathrm{G}$ AT $2 \mathrm{~Hz}$.

\begin{tabular}{|c|c|}
\hline Sample & Specific piezoelectric response [V/g] \\
\hline $\begin{array}{c}\text { Commercial } \\
\text { PVdF film }\end{array}$ & 1.4 \\
\hline $\begin{array}{l}\text { Electrospun } \\
\text { PVdF 6008 }\end{array}$ & 67 \\
\hline $\begin{array}{l}\text { Electrospun } \\
\text { PVdF-TrFe }\end{array}$ & 290 \\
\hline
\end{tabular}

\section{ACKNOWLEDGMENT}

The authors are grateful to Solvay Specialty Polymers for polymers used in this work.

\section{REFERENCES}

[1] S. Ramakrishna, K. Fujihara, W. E. Teo, T. C. Lim, Z. Ma (Eds), "An Introduction to Electrospinning and Nanofibers", World Scientific Publishing Co. Pte. Ltd, NJ, USA 2005.

[2] C. Ribeiro, V. Sencadas, J. L. Gómez Ribelles and S. Lanceros-Méndez, Influence of Processing Conditions on Polymorphism and Nanofiber Morphology of Electroactive Poly(vinylidene fluoride) Electrospun Membranes, Soft Materials 8:3, 274-287, 2010.

[3] Z.-M. Huang, Y.-Z. Zhang, M. Kotaki, S. Ramakrishna, "A review on polymer nanofibers by electrospinning and their applications in nanocomposites", Composites Science and Techn. 63, 2223-2253, 2003.

[4] Z. Dong, S. J. Kennedy, Y. Wu, "Electrospinning materials for energyrelated applications and devices", Journal of Power Sources 196, 48864904, 2011.

[5] H.S. Nalwa (ed.), "Ferroelectric polymers", Marcel Dekker: New York, chapters 1-4, 1995.

[6] J. Gomes, J. Serrado Nunes, V. Sencadas, and S. Lanceros-Mendez, "Influence of the $\beta$-phase content and degree of crystallinity on the piezo- and ferroelectric properties of poly(vinylidene fluoride)," Smart Mater. Struct., vol. 19, p. 065010, 2010.

[7] L. Persano, C. Dagdeviren, Y. Su, Y. Zhang, S. Girardo, D. Pisignano, Y. Huang and J. A. Rogers, "High PerformancePiezoelectric Devices Based on Aligned Arrays of Nanofibers of Poly[(vinylidenefluoride-cotrifluoroethylene]", Nature Comm., vol. 4, Article no: 1633, 2013.

[8] J. Pu, X. Yan, Y. Jiang, C. Chang, L. Lin, "Piezoelectric actuation of direct-write electrospun fibers", Sensors and Actuators A: Physical, Volume 164, Issues 1-2, Pages 131-136, November-December 2010.

[9] M. Zaccaria, D. Fabiani, A. Zucchelli, and J. Belcari, "Electrospinning: A versatile technique for energy storage and sensor applications," in 2014 AEIT Annual Conference 2014, pp. 1-4.

[10] S. Dey, M. Purahmad, S. S. Ray, A. L. Yarin, and M. Dutta, "Investigation of PVDF-TrFE nanofibers for energy harvesting," 2012 IEEE Nanotechnol. Mater. Devices Conf. (NMDC), pp. 21-24, 2012.

[11] C. Chang, V. H. Tran, J. Wang, Y. K. Fuh, and L. Lin, "Direct-write piezoelectric polymeric nanogenerator with high energy conversion efficiency," Nano Lett., vol. 10, pp. 726-731, 2010. 